\title{
Characterizing Dry Electrodes Impedance by Parametric Modeling for Arm Wearable Long-term Cardiac Rhythm Monitoring
}

\author{
Antonio Bosnjak ${ }^{1}$, Pedro Linares ${ }^{2}$, James McLaughlin ${ }^{3}$, Omar J. Escalona ${ }^{3}$ \\ ${ }^{1}$ Centro de Procesamiento de Imágenes. Universidad de Carabobo. Valencia. Venezuela \\ ${ }^{2}$ CEMVICC. Universidad de Carabobo. Valencia. Venezuela \\ ${ }^{3}$ University of Ulster, NIBEC, Newtownabbey, Northern Ireland
}

\begin{abstract}
There are cardiac patients requiring their ECG to be constantly monitored. The quality of long-term electrical activity feature measurements in the ECG may rely considerably on the electrodes and their long-term skin interface performance stability; thus, affecting the accuracy of clinical diagnosis and treatment. Particularly, misbehavior of the skin-electrode impedance $\left(\mathbf{Z}_{S E}\right)$ may cause a large degree of distortion in the ECG signal; therefore, it is important to analyze, characterize and provide a reliable modeling of any emerging dry electrode technology being used for long term ECG monitoring. In this paper we present a useful methodology for modeling the $\boldsymbol{Z}_{S E}$ behavior. With the objective to obtain these parameters, we tested four methods for fitting curves: a) 1-time constant impedance of a circuit. b) 2-time constant impedance measurement. c) A fractional calculus with 1-time constant impedance, and d) A fractional calculus with 2-time constant impedance. As results we obtained that the 2-time constant circuit implementation of $\boldsymbol{Z}_{S E}$ using a fractional calculus proved to be best fitted to the phase plot measured with the Solartron analyzer.
\end{abstract}

\section{Introduction}

Continuous monitoring of heart electrical signals is necessary for a number of cardiac patients due to the characteristics of their particular conditions. An instance of this is the need of implanting loop recorders, by means of a surgical procedure, in cases that require long term rhythm monitoring. This procedure allows for monitoring the patient for a period that can exceed two years. Problems related to this procedure include the high cost of the device and the risks derived from its surgical nature such as anesthetic reactions or infections [1]. The use of wearable devices for physiological measurement purposes has been the focus of growing attention [2]. The study of different electrodes with high performance characteristics is currently an active research area [3][4].
Commonly, electrodes are classified into three broad groups, namely wet (also called gelled), dry and capacitive coupled electrodes. Wearable monitoring systems can conveniently locate sensors in the wrist and arm when aiming at long term studies. In that case, it is also convenient to use dry sensors because they do not require maintaining the gelling of the skin-electrode interface. An inconvenient of dry electrodes is their higher impedance, compared to the gelled ones, however it has been reported that the response to motion artifacts can be comparable [6].

The desirable conditions for electrodes are: a low skin contact impedance, close to zero, and a null contact potential. However, it is not feasible to have real electrodes with these characteristics. The realistic aim is to develop electrodes with low skin-electrode impedance, denoted $Z_{S E}$, and that show similar characteristics to subsequent electrodes in the settings selected for signals recording.

The target of this work is to advance in a method for the characterization of electrodes by means of parametric modeling with particular interest in dry electrodes for arm wearable sensors. This includes the assessment and comparison of $\boldsymbol{Z}_{S E}$ characteristics and frequency response in the $1 \mathrm{~Hz}-100 \mathrm{~Hz}$ range.

\section{Methods}

In this work, we used a set up for the measurements identical to the structured for our previous work [7]. That is, we determined the electrical impedance by means of a Schlumberger SI 1260 impedance/gain-phase analyzer combined with a biological sample interface SOLARTRON 1294 (Solartron 1260 and Solartron 1294, Solartron Analytical, Farnborough, UK). This arrangement has been reported to be reliable and accurate in literature [8].

The "3-electrode" technique used by us to characterize the skin-electrode impedance $\left(\boldsymbol{Z}_{S E}\right)$ in [7] is repeated here. In this scheme, two $\mathrm{Ag} / \mathrm{AgCl}$ electrodes (Ambu, Blue Sensor SP-00-S) are placed in the forearm so that 
they act as reference and current return. The electrode under study was located at $5 \mathrm{~cm}$ from the reference and connected to the yellow and black terminals of the SI 1260 as shown in figure 1.

Physical elements that are circulated by the test current consist of the test electrode, the skin-electrode interface, the epidermis and the dermis. The circuit is closed with the current returning through the dermis, epidermis, gel and the return electrode (skin-electrode interface for the return electrode) [9]. The bottom of figure 1 illustrates the model corresponding elements

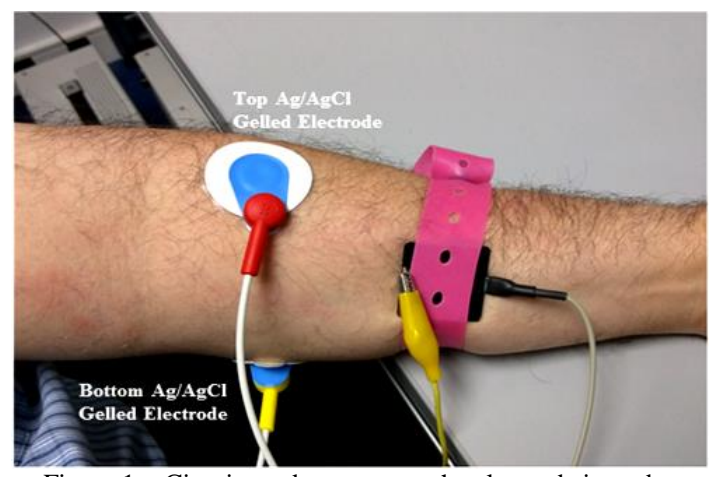

Figure 1. Circuit used to measure the electrode impedance.

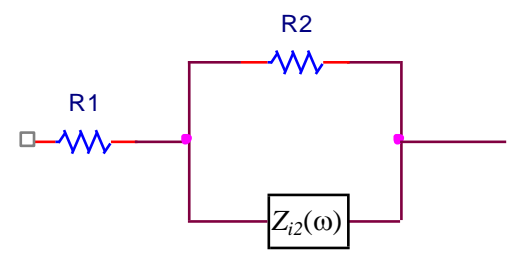

Figure 2. Three element electrical circuit model for electrical-tissue interface.

According to the three elements model of figure 2, $\mathbf{R}_{\mathbf{1}}$ represents the electrolyte intrinsic resistance, $\mathbf{R}_{\mathbf{2}}$ corresponds to the low frequency impedance of the skinelectrode interface. Similarly, $Z_{i 2}(\omega)$ represents the skinelectrode complex impedance. It has been determined that the capacitor impedance theoretical model given by $Z(\omega)=1 / j \omega C$ can be improved for applications in Bioengineering using a fractional calculus approach. Using this technique, the impedance is expressed as: $Z_{i 2}(\omega)=1 /(j \omega)^{\alpha} C_{\beta}$ where $0<\alpha<1$. The parameter $\alpha$ is used in order to adjust the impedance so that it can fit the actual data variability.

If the single time constant model is used, the circuit impedance $\boldsymbol{Z}_{S E}$ in the Laplace domain is expressed by:

$$
Z_{S E}=\frac{R_{1}+R_{2}+s^{\alpha} R_{1} R_{2} C_{\beta 2}}{1+s^{\alpha} R_{2} C_{\beta 2}}
$$

Substituting $s^{\alpha}$ by $(j \omega)^{\alpha}$ and extracting the complex roots of $j$, the solutions are obtained using: $(j \omega)^{\alpha} \rightarrow(\cos \theta+j \sin \theta) \omega^{\alpha}$ and replacing them in equation (1), the following expression is found:

$Z_{S E}=\frac{R_{T}+\omega^{\alpha}\left(2 R_{1} \tau_{2}+R_{2} \tau_{2}\right) \cos \theta+\omega^{2 \alpha} R_{1} \tau_{2}^{2}-j \omega^{\alpha} R_{2} \tau_{2} \sin \theta}{1+2 \omega^{\alpha} \tau_{2} \cos \theta+\omega^{2 \alpha} \tau_{2}^{2}}$

Where:

$$
\tau_{2}=R_{2} C_{\beta 2} \quad \text { and } \quad R_{T}=R_{1}+R_{2}
$$

It is worth noticing that $C_{\beta 2}$ must be quantified in Farad $\cdot(\mathrm{sec})^{-\alpha}$ as units. Therefore, the expression for the angle is:

$$
\varphi(\omega)=\tan ^{-1}\left[\frac{-\omega^{\alpha} R_{2} \tau_{2} \sin \theta}{R_{T}+\left(2 \omega^{\alpha} R_{1} \tau_{2}+\omega^{\alpha} R_{2} \tau_{2}\right) \cos \theta+\omega^{2 \alpha} R_{1} \tau_{2}^{2}}\right] \text { (4) }
$$

We incorporate here a variation that considers that capacitors vary their parameters with frequency as proposed in fractional calculus. The steps for verification include performing the measurements with the analyzer, selecting the mathematical model and adjusting the parameters of this model so that the theoretical curve is adjusted to the data obtained by measurements. This work brings an improvement to our previous results by using fractional calculus in the mathematical model.

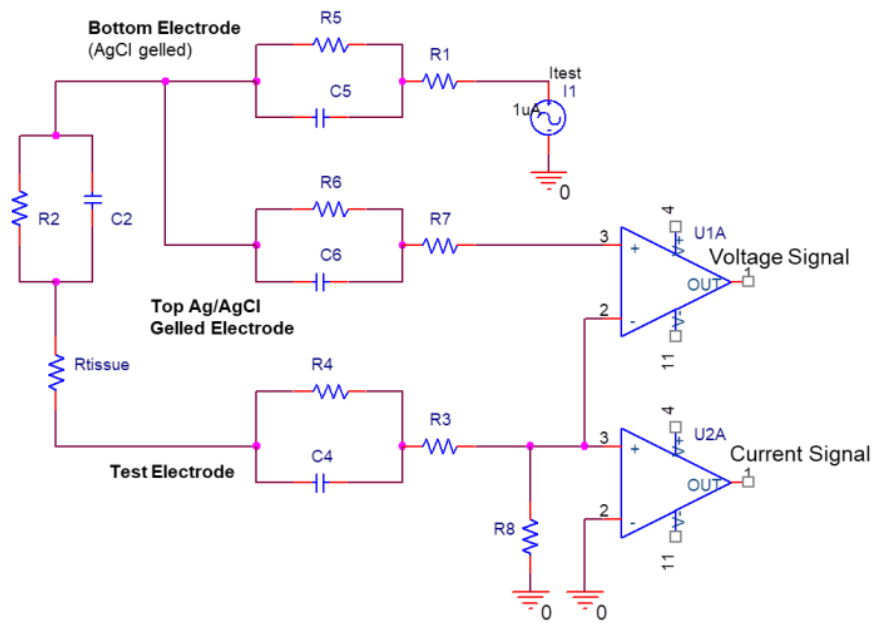

Figure 3. Schematic representation of the circuit setup for measurements assuming a double time constant model.

Further simplifications can be achieved by omitting the parallel elements $\mathrm{R}_{6} / / \mathrm{C}_{6}$, because in the measurement its current is null. The parametric characterization carried out in this work, combines the double-time constant model as proposed in Assambo et al. [10] with the fractional calculus method for biological models presented by Magin and Ovadia in [11] as the approach for solving equations.

The impedance function of the circuit is:

$\frac{V_{1}-V_{2}}{I_{1}}=\frac{R_{2}}{1+s^{\alpha} R_{2} C_{\beta 2}}+R_{\text {tissue }}+\frac{R_{4}}{1+s^{\alpha} R_{4} C_{\beta 4}}+R_{3}$ 
which can be simplified by defining the following resistor:

$$
R_{t 3}=R_{\text {tissue }}+R_{3}
$$

and the following time constants:

$$
\tau_{2}=R_{2} C_{\beta 2} \text { and } \tau_{4}=R_{4} C_{\beta 4}
$$

Once simplified using $R_{t 3}$ the impedance function magnitude can be expressed as:

$$
Z_{S E}=\frac{R_{T}+s^{\alpha}\left[R_{2} \tau_{4}+R_{4} \tau_{2}+R_{t 3}\left(\tau_{2}+\tau_{4}\right)\right]+s^{2 \alpha} \tau_{2} \tau_{4}}{1+s^{\alpha}\left(\tau_{2}+\tau_{4}\right)+s^{2 \alpha} \tau_{2} \tau_{4}}
$$

Where the imaginary impedance is given by:

$$
\begin{aligned}
& \operatorname{Im}\left\{Z_{S E}\right\}=-\omega^{\alpha}\left(R_{2} \tau_{2}+R_{4} \tau_{4}\right) \sin \theta \ldots \\
& -\omega^{2 \varepsilon}\left(R_{2}+R_{4}\right) \tau_{2} \tau_{4} \sin 2 \theta-\omega^{3 \alpha} \tau_{2} \tau_{4}\left[R_{2} \tau_{4}+R_{4} \tau_{2}\right] \sin \theta
\end{aligned}
$$

And the real part of the impedance is given by

$$
\begin{aligned}
& \operatorname{Re}\left\{Z_{S E}\right\}=R_{T}+\omega^{\alpha}\left(R_{2 t} \tau_{2}+R_{4 t} \tau_{4}+2 R_{2} \tau_{4}+2 R_{4} \tau_{2}\right) \cos \theta \ldots \\
& +\omega^{2 \alpha}\left[\left(R_{2}+R_{4}+2 R_{3 t}\right) \tau_{2} \tau_{4}+\left(R_{2}+R_{3 t}\right) \tau_{4}^{2}+\left(R_{4}+R_{3 t}\right) \tau_{2}^{2}\right] . . \\
& +\omega^{2 \alpha}\left[\left(R_{2}+R_{4}+2 R_{3 t}\right) \tau_{2} \tau_{4}\right] \cos 2 \theta \ldots \\
& +\omega^{3 \alpha}\left[R_{2 t} \tau_{2} \tau_{4}^{2}+R_{4 t} \tau_{2}^{2} \tau_{4}\right] \cos \theta+\omega^{4 \alpha} R_{3 t} \tau_{2}^{2} \tau_{4}^{2}
\end{aligned}
$$

Where:

$$
\begin{aligned}
& R_{2 t}=R_{2}+2 R_{3 t} \\
& R_{4 t}=R_{4}+2 R_{3 t} \\
& R_{T}=R_{2}+R_{4}+R_{3 t}
\end{aligned}
$$

The expressions above correspond to an Imaginary and Real part of the transfer function, the phase angle is important because it can be used as a measure of how close the experimental plot, output by the impedance analyzer, and the analytical curve are with respect to each other. Computing the arctangent from equations (9 to 13) the following relation can be obtained:

$$
\varphi(\omega)=\operatorname{Arg}\left(Z_{S E}\right)=\tan ^{-1}\left[\frac{\operatorname{Im}\left\{Z_{S E}\right\}}{\operatorname{Re}\left\{Z_{S E}\right\}}\right]
$$

Thus, these mathematical expressions provide a reference theoretical model, which can be useful for verification, as well as for designing the ECG front-end amplification stage or a required pre-signal processing stage.

A MATLAB program, aiming at the minimization of the least squared error, was developed to adjust parameters of equation (5) to the data obtained experimentally with the Solartron analyzer. This MATLAB script is based on the lsqcurvefit function.

\section{Results}

Five electrodes of different types were measured using the described set up. In figure 4, the selected dry electrodes are shown. In that illustration, the electrodes are numbered as follows: 1) $\mathrm{AgCl}$ stud (Ringtrode,
BQEL1). 2) Iron (Fe), 3) Polyurethane - multipin -A, 4) Polyurethane - multipin -B and 5) Silver disc (Ag).

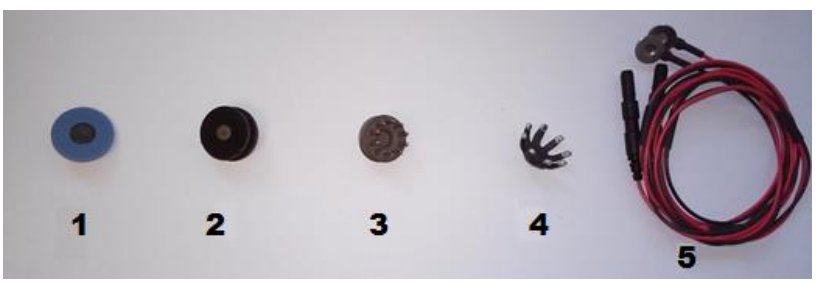

Figure 4. Dry electrodes used for the measurements.

The impedance $\boldsymbol{Z}_{S E}$ was measured for all the electrodes, placing them alternatively over the dry skin, varying frequency in the range from $1 \mathrm{~Hz}$ to $10 \mathrm{kHz}$. In figure 5, a graph corresponding to the measurements is shown. In this initial test, the multi-pin polyurethane-A electrode, had the lowest impedance at any frequency.

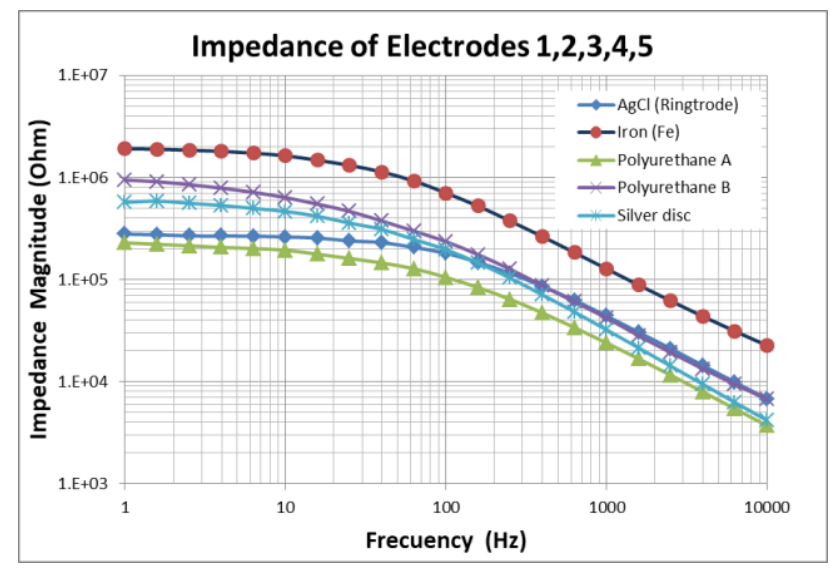

Figure 5. Impedance magnitude vs frequency for the 5 electrodes

Curve fitting tests were performed with measurements from electrodes shown in figure 4. An example of curve fitting for the polyurethane electrode (labelled with number 3) is shown in figure 6. A graph with the curve fitting using a one-time-constant model (red line), together with the adjustment for a double-time-constant model (blue line) are shown. The graph illustrates how, as expected, a circuit including a single discrete capacitor is insufficient to model the electrode under test plus the electrode-skin interface.

It can be seen in figure 6(b), that the method proposed in this work fits the graph with an error lesser than $2 \%$ when fractional calculus is used for estimating the parameters of the electrical elements together with a circuit with two time constants.

Once the minimization is achieved, the values for each of the resistors and capacitors of equations (5 - 14) can be calculated. These values are shown in Table I. 
(a)

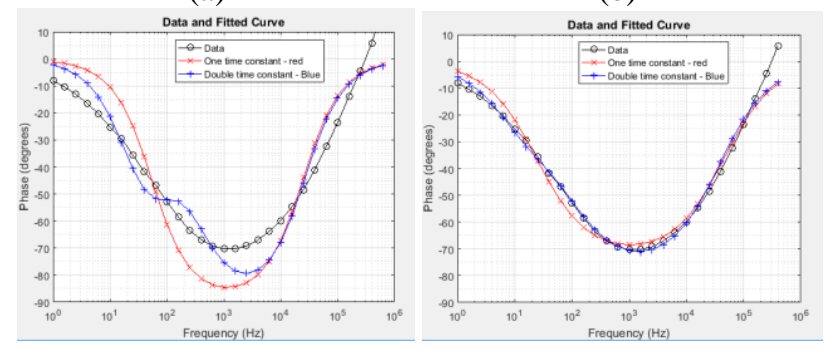

Figure 6(a). Curve fitting using diferential calculus for the phase frequency response. Experimental data ' $o$ '. One-time-constant model ' $x$ ' (red). Double-time-constant ' + ' (Blue). (b) Curve fitting using Fractional calculus proposed on this paper.

TABLE 1. Resulting CirCuit PARAMETERS

\begin{tabular}{c||c||c}
\hline \hline Component & $\begin{array}{c}\text { Polyurethane - multipin } \\
-\boldsymbol{A}\end{array}$ & $\begin{array}{c}\text { Polyurethane - } \\
\text { multipin - } \boldsymbol{B}\end{array}$ \\
\hline $\mathrm{R}_{2}$ & $8,227 \mathrm{k} \Omega$ & $10 \mathrm{k} \Omega$ \\
$\mathrm{C}_{\beta 2}$ & $803,18 n F / \omega^{(1-\alpha)}$ & $616,01 n F / \omega^{(1-\alpha)}$ \\
$\mathrm{R}_{4}$ & $56,28 \mathrm{k} \Omega$ & $20,54 \mathrm{k} \Omega$ \\
$\mathrm{C}_{\beta 4}$ & $544,57 n F / \omega^{(1-\alpha)}$ & $1,5793 \mu F / \omega^{(1-\alpha)}$ \\
$\mathrm{R}_{\mathrm{T}}$ & $66,88 \mathrm{k} \Omega$ & $30,64 \mathrm{k} \Omega$ \\
$\mathrm{Rt} 3$ & $2,372 \mathrm{k} \Omega$ & $99,0 \mathrm{k} \Omega$ \\
ang $\theta$ & $101,4^{\circ}$ & $79,12^{\circ}$ \\
$\alpha$ & 0,5346 & 0,8138 \\
\hline \hline
\end{tabular}

\section{Discussion and Conclusion}

The use of fractional calculus for modeling variations in the skin-electrode interface capacitance with frequency shows a better fitting of theoretical curves to the curves produced with data from measurements. This fact seems to confirm the observations of Magin and Ovidia [11] and encourages further research.

In this study, RC circuit models of double time constant were conveniently adopted in order to approximate the impedance function obtained with the Solartron equipment, for a circuit like that of figure 2 , with discrete parameters, resistances and fractions of capacitors, with the objective to model the electrode material conductivity, the electrode-skin interface and the dermis-epidermis component. This modelling approach is useful for the proper design of the ECG amplifier circuit with a transfer function that would enable a more accurate cardiac ECG signal and reduce linear distortion.

A systematic method for determining the skinelectrode impedances, $\boldsymbol{Z}_{\boldsymbol{S E}}$, has been used to assess a selection of dry electrode materials. Encouraging results were obtained suggesting the development of more adequate mathematical models.

One of the contributions of this work is to show that the skin-electrode interface capacitance and the tissue internal capacitance vary with frequency. This characteristic encourages the use of fractional calculus because it has been shown that it can adjust perfectly the experimental data curves obtained with the Solartron 1260 and 1294 set up. This would enable better characterization of electrodes and their models for more adequate amplifiers design in wearable long term ECG monitoring systems.

\section{Acknowledgements}

This research is supported by funding from the European Union (EU): H2020-MSCA-RISE Programme (WASTCArD Project, Grant \#645759).

\section{References}

[1] W.D. Lynn , O.J. Escalona and D.J. McEneaney, "ECG monitoring techniques using advanced signal recovery and arm worn sensors". IEEE International Conference on Bioinformatics and Biomedicine, IEEE BIBM 2014 , pp. 51-55.

[2] O. Escalona and M. Mendoza, "Electrocardiographic Waveforms Fitness Check Device Technique for Sudden Cardiac Death Risk Screening". IEEE-EMBC 2016, Orlando, FL, pp. 3453-3456.

[3] T. Yilmaz, R. Foster and Y. Hao, "Detecting vital signs with wearable wireless sensors". Sensors. Volume 10, Issue 12, December 2010, pp. 10837-10862

[4] Y.-H Chen, M.O. de Beeck, L. Vanderheyden, et al. "Soft, Comfortable Polymer Dry Electrodes for High Quality ECG and EEG Recording". in Sensors, Volume 14, 2014, pp. 23758-23780.

[5] P. K. Jain, A. K. Tiwari, "Heart monitoring systems-A review," in Computers in Biology and Medicine, Volume 54, 2014, pp. 113

[6] A. Searle and L. Kirkup,, "A direct comparison of wet, dry and insulating bioelectric recording electrodes." in Physiological Measu-rement, Volume 21, 2000, pp. 271-283.

[7] A. Bosnjak, A. Kenneddy, P. Linares, M. Borges, J. McLaughlin, O. Escalona. "Performance assessment of dry electrodes for wearable long term cardiac rhythm monitoring: skin-electrode impedance spectroscopy". Proc. 39th Annual International Conference of the IEEE Engineering and Medicine Biology Society. Jeju Island, South Korea. July 2017.

[8] J. Strand-Amundsen, C. Tronstad, H. Kalvøy, et al. "In vivo characterization of ischemic small intestine using bioimpedance measurements". Physiological Measurement, vol. 37, Issue 2, pp. 257-275. January 2016.

[9] E. McAdams, "Bioelectrodes". Encyclopedia of Medical Devices and Instrumentation, Second Edition, edited by John G. Webster. Copyright $\odot 2006$ John Wiley \& Sons, Inc. pp. $120-166$.

[10] C. Assambo, R. Dozio, A. Baba and M. J. Burke "Determination of the Parameters of the Skin Electrode Impedance Model for ECG Measurement". Proc. 6th Int. Conf. on Electronics, Hardware, Wireless and Optical Communications, Corfu, February 2007, pp. 540-318.

[11] R. L. Magin, M. Ovadia. "Modeling the Cardiac Tissue Electrode Interface Using Fractional Calculus". Journal of Vibration and Control, () 2008 SAGE Publications. Volume 14, pp. 1431 - 1442.

Address for correspondence.

Name: Omar J. Escalona

Full postal address: Ulster University, Shore Road, Newtownabbey, BT37 0QB, UK.

E-mail address: oj.escalona@ulster.ac.uk 